\title{
Influence of a defect particle on the structure of a classical two-dimensional cluster
}

\author{
K. Nelissen, B. Partoens, ${ }^{*}$ and F. M. Peeters ${ }^{\dagger}$ \\ Departement Fysica, Universiteit Antwerpen (Campus Drie Eiken), Universiteitsplein 1, B-2610 Antwerpen, Belgium
}

(Received 24 November 2003; published 16 April 2004)

\begin{abstract}
A system of classical charged particles interacting through a Coulomb repulsive potential which are confined in a two-dimensional parabolic trap is studied. We allow one or two particles, called defect particles, to have a different mass and/or charge than the other particles. The structure of the whole system depends on the mass and the charge of the defects and the total number of particles in the system. The ground state configurations are investigated and phase diagrams are constructed, which explain the recent experimental results of Grzybowski et al. [Phys. Rev. E 64, 11603 (2001)]. We found that several of the experimental configurations are metastable and that replacing the Coulomb interparticle potential by an inversely quadratic one has only a minor effect on the results.
\end{abstract}

DOI: 10.1103/PhysRevE.69.046605

PACS number(s): 45.20. $-\mathrm{d}, 45.05 .+\mathrm{x}, 61.46 .+\mathrm{w}$

\section{INTRODUCTION}

Classical clusters of repulsive particles confined in traps are both theoretically and experimentally a study object for many decades due to their applicability to a wide variety of systems. These systems are composed of a finite number of charged classical particles which can move in a twodimensional (2D) plane and are confined in the plane by an external applied potential. They are the classical analog of the 2D quantum dot. Typical experimental realizations of such systems are electrons on the surface of liquid helium [1], plasmas with dust particles [2], ion traps [3], vortices in superfluids [4], confined ferromagnetic particles [5], charged metallic balls above a plane conductor [6], and colloidal crystals [7]. The 2D charged clusters also resemble the problem of charge distribution studied by Mayer [8] and by Thomson in his "plum-pudding" model of the atom $[9,10]$.

When the particles are confined to a parabolic trap, there is a competition between the bulk triangular lattice and the circular confinement potential that tries to force the charged particles into ringlike configurations. Those configurations were systematically studied in Ref. [11] and a Mendeleevtype of table for these classical atomlike structures was constructed. The spectral properties of the ground state configurations were presented in Refs. $[12,13]$ and generalized to screened Coulomb [14,15] and logarithmic [10,14,16,17] interparticle interactions.

Recently, an experiment [18] was performed where the self-assembly of millimeter-sized, magnetized disks floating on a liquid-air interface, and rotating under the influence of a rotating external magnetic field was considered. Spinning of the disks results in a hydrodynamic repulsion between them, while the rotating magnetic field produces an average confining potential acting on all disks. The interplay between hydrodynamic and magnetic interactions between the disks leads to the formation of ordered patterns, which shows that it can be considered as a dynamic self-assembling system.

\footnotetext{
*Electronic address: bart.partoens@ua.ac.be

†Electronic address: francois.peeters@ua.ac.be
}

All the disks in this experiment were identical, except for one or two which had a larger radius than the others. These results inspired us to extend our previous work on classical systems to systems with one or two defects and to see if the obtained static configurations resemble the experimental dynamic self-assembled ordered structures.

The same authors modeled this system by integrating the equations of motion which account for the hydrodynamic and magnetic forces acting in the system [19]. The relative value of the parameters characterizing these interactions were deduced from the experiment. Results for identical disks were reported and for a few configurations with up to seven small disks plus a large one. The simulated structures reproduce the patterns observed experimentally.

The structure and melting of clusters consisting of twospecies of charged particles with the same mass were studied theoretically in Ref. [20]. The system is similar to the one studied, where a fraction of the particles had a charge twice as large as the others. The particles were found to arrange themselves in rings, where those with a larger charge were expelled to the edge of the trap.

Here we extend the works of Refs. $[19,20]$ and show that allowing the "defect" particles to have a different mass and/or charge dramatically modifies the obtained configurations. We found that the defect particles can reside anywhere in the cluster depending on their mass and charge. The obtained results will be summarized in phase diagrams. We also found that the introduction of the defect particle leads to an increase in the number of metastable states, some of which were observed in the experiment of Ref. [18].

The paper is organized as follows. In Sec. II our model system is introduced. The results for one and two defects are shown in Secs. III and IV, respectively. Also, in these sections a comparison with the experimental results of Ref. [18] is given. Our conclusions are presented in Sec. V.

\section{MODEL SYSTEM}

In Ref. [18] an empirical Hamiltonian for the experimental system was proposed, namely, 


$$
H=\sum_{i} c_{1}(H, M) r_{i}^{2}+\sum_{i<j} c_{2}\left(\omega, a_{1}, a_{2}, \rho\right) \frac{1}{\left|\mathbf{r}_{i}-\mathbf{r}_{j}\right|^{2}} .
$$

The first term is the confinement potential and is due to the rotating magnet. The confinement strength $c_{1}$ depends on the magnetization $M$ of the magnet and its distance $H$ from the interface. The second term describes the hydrodynamic repulsion between the disks. The coefficient $c_{2}$ depends on the angular velocity $\omega$ of the magnet, the radii of the particles $a_{1}$ and the $\operatorname{defect}(\mathrm{s}) a_{2}$, and the density $\rho$.

Motivated by this empirical Hamiltonian, we consider a system with $N$ point-like classical particles interacting through a more general $1 / r^{n}$ potential. The particles are free to move in two dimensions but are confined by a parabolic trap centered at the origin. The general Hamiltonian of the system for particles with different masses and charges is

$$
H=\frac{1}{2} \sum_{i=1}^{N} m_{i}^{*} \omega_{0}^{2} r_{i}^{2}+\frac{1}{\epsilon} \sum_{i<j}^{N} \frac{q_{i}^{*} q_{j}^{*}}{\left|\mathbf{r}_{i}-\mathbf{r}_{j}\right|^{n}},
$$

where $m_{i}^{*}$ is the effective mass of the particle and $q_{i}^{*}$ its charge, $\omega_{0}$ the radial confinement frequency, and $\epsilon$ the dielectric constant of the medium the particles are moving in. The Hamiltonian can be transformed to a dimensionless form using the following units: $r_{0}=\left(2 Q^{2} / M \epsilon \omega_{0}^{2}\right)^{1 /(2+n)}$ for the length and $E_{0}=M \omega_{0}^{2} r_{0}^{2} / 2$ for the energy. $M$ and $Q$ represent the reference mass and charge. After rescaling we can write the Hamiltonian in dimensionless form

$$
H=\sum_{i=1}^{N} m_{i} r_{i}^{2}+\sum_{i>j}^{N} \frac{q_{i} q_{j}}{\left|\mathbf{r}_{i}-\mathbf{r}_{j}\right|^{n}}
$$

with $m_{i}=m_{i}^{*} / M$ and $q_{i}=q_{i}^{*} / Q$, respectively, the rescaled mass and charge of particle $i$. We take $m_{i}=1$ and $q_{i}=1$ for all particles except one or two, which we call further the defect(s). In the rest of the article, we always refer with $m$ and $q$ to the mass and charge of the defect particle(s).

To find the minimum energy configuration we used the Monte Carlo simulation technique extended with a Newton optimalization technique, as first used in Ref. [12]. This last technique increases the convergence and the accuracy of the local minimum. In order to be sure to have found the ground state configuration, one has to run the Monte Carlo simulation routine many times, starting with a different initial random configuration. To be sure that the obtained configuration is a stable one, each particle was moved around its "equilibrium" position and the force on the particle and the energy of the configuration was monitored.

\section{SYSTEMS WITH ONE DEFECT}

The interparticle potential was taken to be Coulombic [i.e., $n=1$ in Eq. (2)]. The stable configurations were studied as functions of the charge and mass of the defect particle which was embedded in a system of $N$ identical particles. The results for the ground state configuration are summarized in phase diagrams, which for $N=3, \ldots, 7$ are shown in Figs. 1(a)-1(e). The obtained ground state configurations can be classified into three groups: (i) closed ring configurations
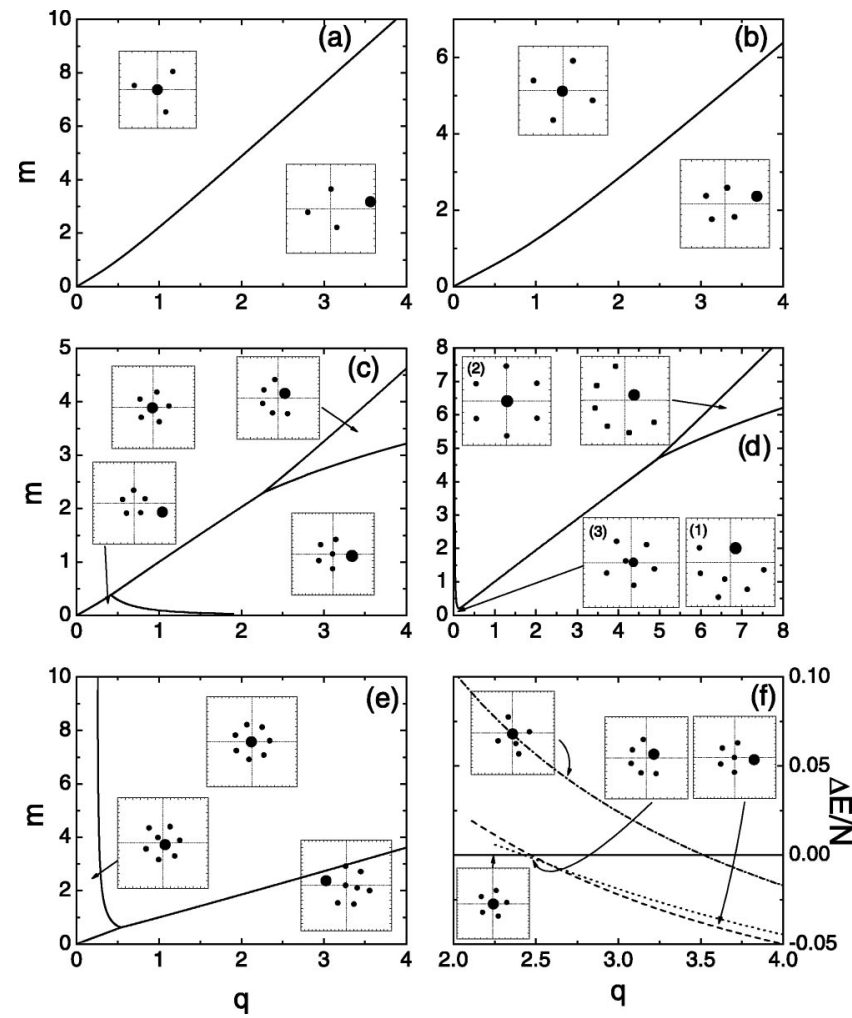

FIG. 1. (a)-(e) Phase diagrams for the ground state configuration of a classical cluster with $N=3, \ldots, 7$ particles and a single defect particle. The particles interact through the Coulomb potential [i.e., $n=1$ in Eq. (2)]. The insets show the typical configurations in each region of the phase diagram. (f) The energy difference of all stable configurations with the energy of the closed ring configuration as a function of the charge of the defect for $N=5$ particles and fixed $m$ $=2.4$.

with the defect in the center and the other particles forming a ring around it, which is realized in the upper left part $(m$ $\gg q$ ) of the phase diagram, (ii) open ring configurations in which the outer ring around the defect is not closed, and (iii) cluster configurations in which the defect is not situated in the center and the other particles are grouped in a cluster opposite to the position of the defect, as realized in the lower right part $(m \ll q)$ of the phase diagram.

One can see that the complexity of the phase diagrams increases, in general, with the number of particles. However, there is always a closed ring configuration and a cluster configuration. A closed ring configuration is the ground state when the defect has a large mass. The defect with a large mass can lower its confinement energy by moving to the center of the trap. However, when the charge becomes large, the repulsive interparticle interaction energy increases, which is minimized when the particle is situated at the edge of the system, which leads to the formation of a cluster configuration. This competition between the confinement energy and the interparticle energy determines the ground state. Notice that the phase boundary between both phases is almost linear and the slope of this boundary decreases with increasing number of particles. This means that, when we start from a system in a ground state cluster configuration, putting an 


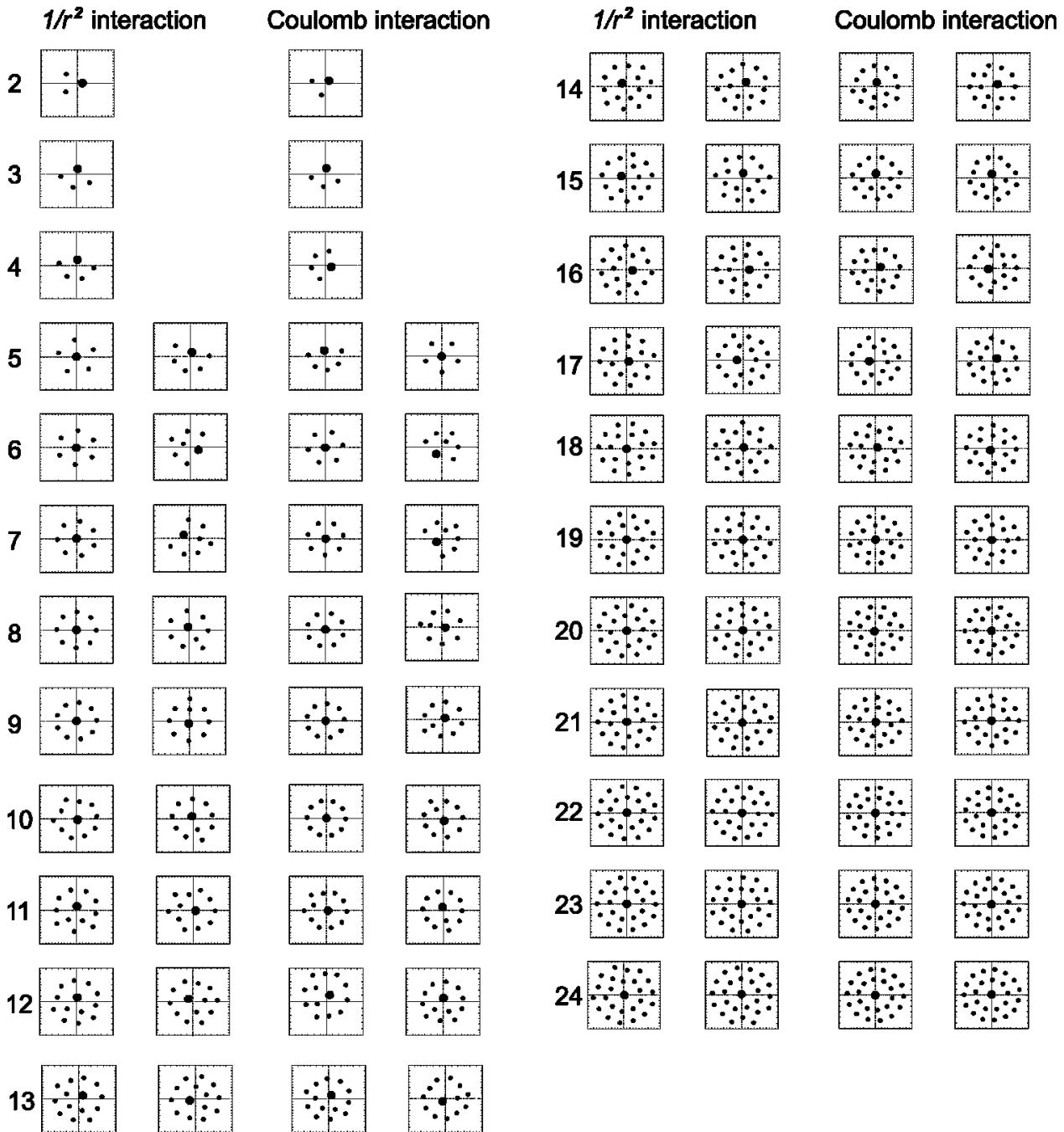

FIG. 2. The ground state and first metastable configurations for one defect and $N=2, \ldots, 24$ particles. Results are shown for $1 / r^{2}$ and Coulomb interaction potentials. The mass and charge of the defect particle is fixed to $m=4$ and $q=4.4$. extra particle will move the defect even further away from the center in order to minimize the Coulomb repulsive energy. This expends extra confinement energy, which can lead to a transition from a cluster configuration to a closed ring configuration with the defect in the center.

The phase diagrams only show the ground state configurations, but several metastable configurations can be realized at the same time. An example is shown in Fig. 1(f), where the energy difference of all stable configurations with respect to the closed ring configuration for five particles and a single defect is plotted as a function of the defect charge for fixed $m=2.4$. Notice that: (i) configurations which are the ground state in a certain region can still be a metastable state outside that region, and (ii) stable configurations can be realized which never become the ground state.

Notice that in the system with five particles and one defect, four ground state configurations are possible, while for the six particle system only three configurations exist. However, for small values of the defect charge, the cluster configuration is also realized but only in a very narrow region. This can be understood as follows. If one starts from the cluster configuration with a large defect charge [see inset (1) in Fig. 1(d)], and diminishes the charge slowly, the defect will move closer to the center [see inset (3) in Fig. 1(d)]. At some point the cluster configuration will become a metastable configuration and the closed ring configuration [see inset (2) in Fig. 1(d)] will take over as the ground state. When the defect charge becomes very small, the defect sits almost in the center, close to the central particle [see inset (3) in Fig. 1(d)]. These two particles will now act as one effective particle. We call this still a cluster configuration because it can be obtained in a continuous way from the cluster configuration by changing the defect charge $q$. From previous work [11] we know that the ground state configuration of six identical particles is $(1,5)$ (one particle in the center with a ring of five particles around it), and consequently the cluster configuration becomes the ground state again.

Also in the phase diagram for seven particles and one defect, one can see this small $q$ phase but now in a larger part of the $(m, q)$ plane. Again, the central particle and the defect behave as a single particle with almost unit charge. However, an extra phase exists in comparison with the six particle system, because the ground state configuration for a large defect charge has two central particles, while the ground state configuration for seven identical particles (which is realized for very small defect charges) has only one central particle.

We compared the results of our simulations with the recent experimental results of Ref. [18]. Although this experi- 
2

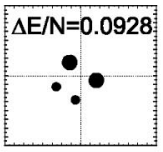

3

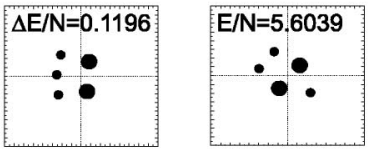

4

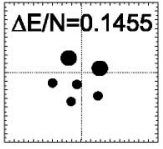

5

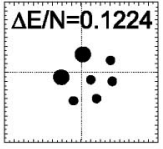

6

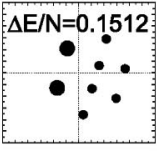

7

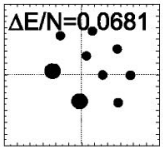

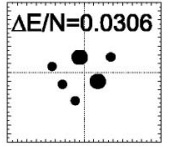
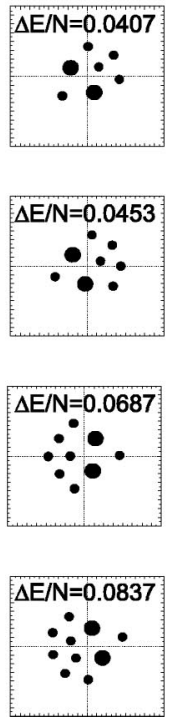
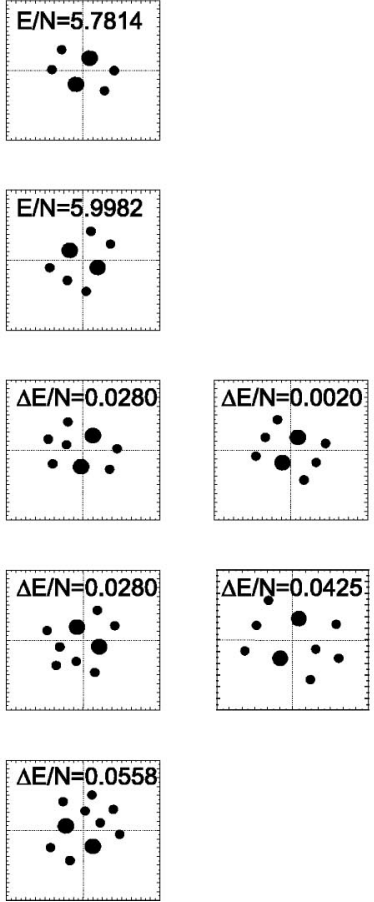
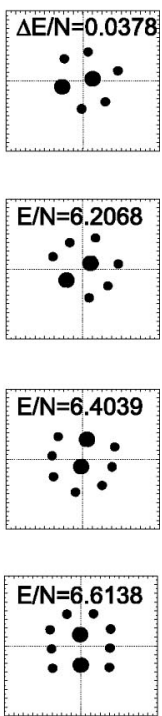

FIG. 3. The ground state and first metastable configurations for two defects and $N=2, \ldots, 8$ particles. Results are shown for a $1 / r^{2}$ interaction potential. The mass and charge of the defect particle is fixed to $m=4$ and $q=4.4$. For the ground state the energy per particle is given, and for the metastable states the energy difference with the ground state is given. ment describes a dynamical system, we were able to reproduce the experimentally observed configurations with our static theory. As it is nontrivial to derive from the experiment the appropriate values for $m$ and $q$, we had to make a good choice. In the experiment, slightly different configurations were also observed for different disk diameters and angular velocities of the magnet. Therefore, a one-to-one agreement with the experiment is impossible. However, the best qualitative agreement was obtained for $m=4$ and $q=4.4$, which we fixed in all subsequent comparisons with the experiment of Ref. [18]. Figure 2 shows the ground state configuration and the first metastable state for systems with 2 up to 24 particles (which was also the largest system in the experiment) for Coulomb interaction and for a $1 / r^{2}$ repulsive interaction between the particles. A good qualitative agreement with the configurations shown in Figs. 4 and 5 of Ref. [18] is obtained. As already mentioned in Sec. II, in Ref. [18] it is argued that the interaction potential between the disks is of the form $1 / r^{2}$. However, one can notice that there are only minor differences between the configurations for a $1 / r^{2}$ interaction and a Coulomb interaction (after a rotation of one of the configurations around the origin which does not change its energy).

From the empirical Hamiltonian (1) one can see that the confining strength $c_{1}$ only depends on the magnetization $M$ of the magnet and its distance $H$ from the interface, and is thus equal for all disks, including the one with the larger diameter. In our model this would result in an equal mass for all particles. We want to stress that both $m$ and $q$ had to differ from 1 in order to be able to reproduce the experimental configurations. However, we believe that the empirical Hamiltonian must be slightly changed. The confinement force of each disk in the experiment depends on its magnetic moment. The magnetic moment of a disk is proportional to its volume, and thus it depends on the square of the disk radius, $a^{2}$. Therefore, the coefficient $c_{1}$ in the empirical Hamiltonian has also to depend on $a$, in contrast to what was claimed in Ref. [18].

From their experimental results, the authors deduce six qualitative rules (written in italic below) which describe the formation of the different patterns. We will comment on each of them using our theoretical results.

(1) The largest disk (i.e., a defect particle) occupies the central position on the axis of rotation of the magnet. From the phase diagrams in Fig. 1 we know that only when the mass of the defect is large in comparison with its charge, the defect will occupy the central position. Therefore, changing some experimental parameters like the disk radius or the angular velocity of the magnet may lead to a different configuration. For example, as $c_{1}$ depends on $a$, decreasing the radius of the largest disk may lead to a cluster configuration.

(2) Smaller disks (i.e., the identical particles) organize 
into shells around the large disk (i.e., the defect particle). The formation of shells is also clearly observed in our numerical results.

(3) The larger the size difference between the defect and the other disks, the simpler the morphology of the aggregates. As $c_{1}$ depends on $a$, increasing the size difference corresponds approximately with increasing the mass of the defect particle. In the phase diagrams this means that one moves upwards (away from the linear phase boundary). It is clear from our phase diagrams (Fig. 1) that this leads to simpler configurations.

(4) Shells are populated consecutively, i.e., the second shell fills, only after the first shell is completed. From our theoretical results we can indeed deduce that the first shell is populated with up to ten particles before the second shell is formed. However, from Fig. 2 one can see that the formation of the second shell starts with particles positioned between the defect and the outer ring and a clear distinction between the first and second shell is not always possible. Around 18-19 particles and beyond, a closed second shell is formed.

(5) When the size of an atom (i.e., the whole system) is not too large, the occupancies of the shells can be predicted by simple geometric considerations (polygon method). We want to stress that the polygon method gives only the correct configuration for certain systems and that for systems with a small number of particles, even open ring configurations are observed. The polygon method will work better if the closed ring configuration is the ground state. Experimentally, this can be achieved by increasing the radius of the defect particle.

(6) Closed ring structures are more stable than open ring structures. We found that the stability regions for openshell configurations are indeed smaller than the region in which the closed ring structure is the ground state.

All configurations obtained theoretically in Ref. [19] (one up to seven disks and one defect) can also be found in the phase diagrams of Fig. 1.

\section{SYSTEMS WITH TWO DEFECTS}

Experimental results were also reported in Ref. [18] for systems containing two defects. For our simulations with two defects we used the same values for $m=4$ and $q=4.4$ as before. We reproduced most of the experimental configurations by our simulations. In the experimental paper, Ref. [18], several stable configurations were found. Our numerical results shown in Fig. 3 must be compared with the first seven rows in Fig. 6 of Ref. [18], which match exactly with these experimental results. The particles organize into groups on either side of the axis joining the defect particles. All possible combinations are allowed (i.e. $n$ particles on one side of the axis joining the two defect particles and $N-n$ on the other side), provided that the number of particles in one group does not exceed seven. Note indeed that for the system with eight particles, experimentally as well as theoretically, always at least one particle is situated at each side of this line. Not all numerically obtained ground states for five up to eight particles were observed experimentally. Those corresponding to the open ring configurations, which are shown in

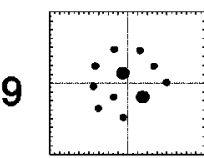

10

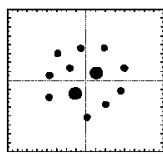

11

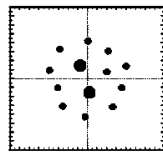

12

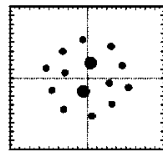

13

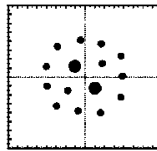

14

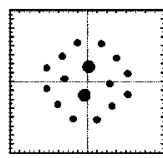

15

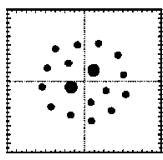

16

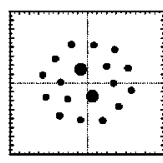

17

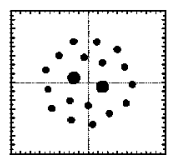

18

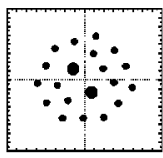

27

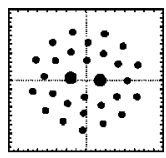

19

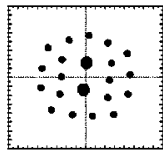

28

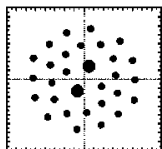

20

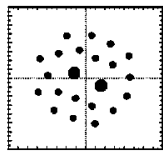

29

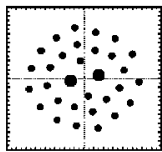

21

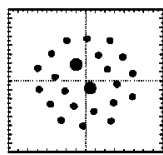

30

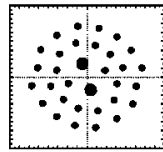

22

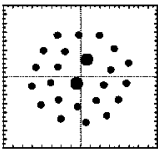

31

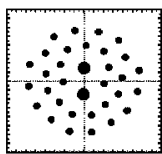

23

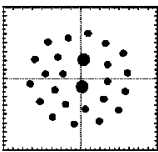

32

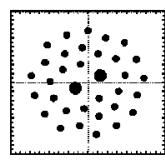

FIG. 4. The ground state configurations for two defects and $N$ $=9, \ldots, 32$ particles. Results are shown for a $1 / r^{2}$ interaction potential. The mass and charge of the defect particle is fixed to $m=4$ and $q=4.4$.

the right column of Fig. 3, were not reported in Ref. [18]. It is not obvious why these ground state configurations were not reported in Ref. [18] and why the experimental systems seem to be trapped into a metastable configuration.

The numerical configurations from nine up to 32 particles and two defects are shown in Fig. 4 and should be compared with the results shown in Figs. 6 and 7 of Ref. [18]. Experimentally, the last open ring configuration is found for 11 small disks, while numerically it is found for ten particles. Experimentally, starting from 12 disks, many polymorphic configurations were observed and only representative ex- 
amples are shown. Also, numerically many metastable configurations were found with very similar structures, which, given the limited amount of space, are not reported here.

\section{CONCLUSIONS}

The ground state structure of classical identical pointlike particles with one or two defect particles with a different mass and charge, interacting through a Coulomb or $1 / r^{2}$ potential and confined in a parabolic potential, was studied. We showed that in the presence of one defect particle the ground state depends strongly on the charge and mass of the defect particle: closed ring, open ring, and cluster configurations are found. Phase diagrams were constructed.
A good qualitative agreement was obtained with recent experimental results [18] for systems with both one and two defects. It is interesting to note that the experimental parameters were chosen rather nicely in a region of the phase diagram with many possible states, namely close to the phase boundary between the closed ring and cluster configuration. The mass and charge of the defect particle were taken the same in all our simulations in order to explain all the obtained configurations.

\section{ACKNOWLEDGMENTS}

This work was supported by the Flemish Science Foundation and the EU-RTN on "Surface Electrons."
[1] P. Leiderer, W. Ebner, and V. B. Shikin, Surf. Sci. 113, 405 (1992).

[2] J. H. Chu and Lin I, Phys. Rev. Lett. 72, 4009 (1994).

[3] B. G. Levi, Phys. Today 41, 17 (1988).

[4] Y. Kondo, J. S. Korhonen, M. Krusius, V. V. Dmitriev, E. V. Thuneberg, and G. E. Volovik, Phys. Rev. Lett. 68, 3331 (1992).

[5] M. Golosovsky, Y. Saado, and D. Davidov, Phys. Rev. E 65, 061405 (2002).

[6] M. Saint Jean, C. Even, and C. Guthmann, Europhys. Lett. 55, 45 (2001)

[7] R. Bubeck, C. Bechinger, S. Neser, and P. Leiderer, Phys. Rev. Lett. 82, 3364 (1999).

[8] A. M. Mayer, Nature (London) 18, 258 (1878).

[9] J. J. Thomson, Philos. Mag. 39, 237 (1904).

[10] B. Partoens and F. M. Peeters, J. Phys.: Condens. Matter 9, 5383 (1997).

[11] V. M. Bedanov and F. M. Peeters, Phys. Rev. B 49, 2667
(1994)

[12] V. A. Schweigert and F. M. Peeters, Phys. Rev. B 51, 7700 (1995).

[13] F. M. Peeters, V. A. Schweigert, and V. M. Bedanov, Physica B 212, 237 1995).

[14] Y.-J. Lai and Lin I, Phys. Rev. E 60, 4743 1999).

[15] L. Cândido, J. P. Rino, N. Studart, and F. M. Peeters, J. Phys.: Condens. Matter 10, 11627 (1998).

[16] L. J. Campbell and R. M. Ziff, Phys. Rev. B 20, 1886 (1979).

[17] P. Cheung, M. F. Choi, and P. M. Hui, Solid State Commun. 103, 357 (1997).

[18] B. A. Grzybowski, X. Jiang, H. A. Stone, and G. M. Whitesides, Phys. Rev. E 64, 11603 (2001).

[19] B. A. Grzybowski, H. A. Stone, and G. M. Whitesides, Proc. Natl. Acad. Sci. U.S.A. 7, 4147 (2002).

[20] J. A. Drocco, C. J. Olson Reichhardt, C. Reichhardt, and B. Jankó, Phys. Rev. E 68, 060401 (2003). 